\title{
Effects of Intense Pulsed Light on Tear Film TGF- $\beta$ and Microbiome in Ocular Rosacea with Dry Eye
}

This article was published in the following Dove Press journal:

Clinical Ophthalmology

\author{
Samantha Sagaser (D) \\ Richard Butterfield (D) ${ }^{2}$ \\ Heidi Kosiorek ${ }^{2}$ \\ Yael Kusne $\mathbb{D}^{3}$ \\ Juan Maldonado $\mathbb{1 D}^{4,5}$ \\ Michael P Fautsch (D ${ }^{6}$ \\ Dharmendra Patel ${ }^{3}$ \\ Joanne F Shen (D) $^{3}$ \\ 'Mayo Clinic Alix School of Medicine, \\ Scottsdale, AZ, USA; ${ }^{2}$ Research \\ Biostatistics, Mayo Clinic, Scottsdale, AZ, \\ USA; ${ }^{3}$ Ophthalmology, Mayo Clinic, \\ Scottsdale, AZ, USA; ${ }^{4}$ Knowledge \\ Enterprise, Genomics Core, Arizona \\ State University, Tempe, AZ, USA; \\ ${ }^{5}$ Center for Fundamental and Applied \\ Microbiomics, The Biodesign Institute, \\ Arizona State University, Tempe, AZ, \\ USA; ${ }^{6}$ Ophthalmology, Mayo Clinic, \\ Rochester, MN, USA
}

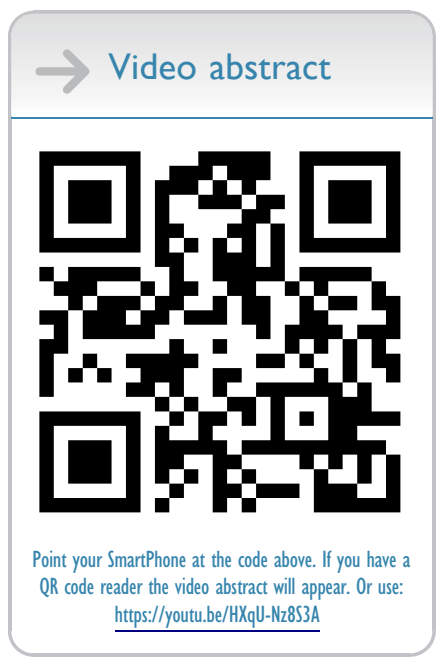

Correspondence: Joanne F Shen Mayo Clinic, 13400 East Shea Blvd, Scottsdale, AZ 85259, USA

Email Shen.Joanne@mayo.edu
Purpose: To assess tear film transforming growth factor-beta (TGF- $\beta$ ) and ocular microbiome changes after intense pulsed light with meibomian gland expression (IPL-MGX) vs only MGX in treating ocular rosacea with dry eye symptoms.

Methods: Twenty patients were randomly assigned to IPL-MGX or MGX. Patients were examined, treated, and administered the ocular surface disease index (OSDI) survey every 4-6 weeks for four total treatments. Tear film and conjunctival samples were collected at first and last visits, and analyzed for TGF- $\beta$ concentration and 16 s rRNA amplicon sequencing of ocular microbiome. Wilcoxon Rank Sum and Sign-Rank were used to examine changes from baseline.

Results: OSDI revealed significantly greater improvement in symptoms after IPL-MGX $(\mathrm{p}=0.030)$ compared to MGX. There was no significant difference in mean TGF- $\beta 1,2$, or 3 concentration after IPL-MGX ( $\mathrm{p}=0.385,0.709,0.948$, respectively). Quantities of Clostridium, Klebsiella, Brevibacterium, Lactobacillus, Neisseria, Streptococcus, Corynebacterium, Butyricicoccus, and Actinomyces were significantly reduced from baseline in both groups but without a significant difference between the two treatment groups.

Conclusion: IPL-MGX improved dry eye symptoms more than MGX alone. IPL treatment offered no additional benefit to MGX in decreasing virulent bacteria present on the ocular surface and did not influence TGF- $\beta$ levels in tears. Prospective studies on IPL-MGX with larger sample sizes are needed to further investigate cytokines and IPL in patients suffering from ocular rosacea with dry eye symptoms.

ClinicalTrials.gov Identifier: NCT03194698.

Keywords: intense pulsed light, IPL, meibomian gland expression, dry eye disease, meibomian gland disorder, ocular rosacea, transforming growth factor-beta, TGF- $\beta$, tear cytokines, ocular microbiome, OSDI; ocular surface disease index

\section{Introduction}

Dry eye disease (DED) is a common condition causing ocular discomfort, reduced visual acuity, and impaired quality of life for as many as $12 \%$ of individuals over the age of $50 .{ }^{1}$ Meibomian glands, modified sebaceous glands that line the upper and lower eyelid margins, secrete the lipid component of tears called meibum. ${ }^{2}$ This lipid meibum keeps the tear film from evaporating off of the ocular surface. ${ }^{3}$ Meibomian gland dysfunction (MGD) is a chronic abnormality of the meibomian glands characterized by duct obstruction and/or changes in the glandular secretions, ${ }^{4,5}$ and is the most common cause of dry eye disease. ${ }^{6}$ In MGD, abnormal meibum obstructs meibomian glands and causes altered tear film that can result in symptoms of eye irritation, ocular surface disease, inflammation, and bacterial 
overgrowth. ${ }^{5,7}$ When MGD is treated early, there is potential for the return of gland function. However, once meibomian glands have atrophied, the patient must live with a decreased quantity of meibum-producing glands for the remainder of life. Despite the variety of treatment options available, patients are often disappointed with the results and continue to experience discomfort. Many of the current therapies only treat the symptoms of DED, rather than addressing the underlying pathophysiology of MGD.

The goal of MGD therapy is to improve the quality and flow of meibum, stabilize tear film, and decrease inflammation. Commonly used therapies include artificial tears, lid margin exfoliation, warm compresses, topical or oral antibiotics, intraductal probing, forced meibomian gland expression (MGX), and automated vectored thermal pulsation. MGX is a relatively inexpensive and readily available treatment option that requires minimal equipment and has proven efficacious in dry eye patients. However, it is often painful and requires frequent treatment sessions. ${ }^{6}$ Despite the variety of therapeutic approaches available for DED, patients are often disappointed with symptom control and continue to have refractory disease.

\section{Intense Pulsed Light}

Intense pulsed light (IPL), a common treatment for dermatological conditions like acne rosacea and hyperpigmentation, is a recognized treatment for ocular rosacea (an inflammatory condition of the eye that can occur in the presence or absence of cutaneous rosacea) with dry eye symptoms. ${ }^{6}$ IPL therapy utilizes a high-intensity noncoherent light in the wavelength range of 500-1200 nm. ${ }^{8}$ It has been an effective treatment for many dermatological conditions as it demonstrates angiodestructive and antiinflammatory properties that prove beneficial for erythema and swelling. In 2002, Dr. Rolando Toyos was the first clinician to suggest the utilization of IPL for ocular rosacea with MGD and DED after coincidentally observing improvement in dry eye signs and symptoms in his patients receiving IPL treatment for facial rejuvenation. ${ }^{9}$ Since then, IPL has emerged as a promising treatment for patients suffering from ocular rosacea with refractory dry eye, and has been a popular topic of research over the past decade. Several studies retrospectively demonstrated efficacy and safety of IPL in ocular rosacea with dry eye when combined with MGX. ${ }^{9-15}$ More recent prospective trials have investigated masked IPL alone, and reported significant improvements in both symptoms and clinical measurements of dry eye. ${ }^{16-18}$ IPL treatment has been accepted in published dry eye protocols such as CEDARS $^{19}$ and TFOS DEWS II. ${ }^{6}$ Despite the growing acceptance of IPL in ophthalmology, the mechanism by which it improves dry eye symptoms is not fully understood.

There are several hypotheses suggesting the various mechanisms of IPL's efficacy in treating ocular rosacea with dry eye. It is thought that the superficial blood vessels in ocular rosacea are the primary site of IPL activity. The general understanding is that intense light is absorbed by chromophores in oxyhemoglobin, causing abnormal blood vessels in inflamed areas to thrombose. ${ }^{7}$ Once closed, these abnormal blood vessels are no longer able to leak inflammatory mediators into the surrounding tissue. In addition, the temporary thermal effect of the light on meibomian glands is thought to decrease the viscosity of meibum, thus improving secretions and tear film quality. ${ }^{12}$ Despite the logic of these ideas, scientific evidence of a mechanism of action is lacking. Identifying a definitive mechanism of action would enable providers to better understand which patients are likely to see improvement in dry eye with IPL treatment. Understanding the physiological changes brought on by IPL may also provide advancement in the understanding of ocular rosacea and MGD, opening doors for future therapeutic interventions.

\section{TGF- $\beta$ Tear Cytokine}

Cytokines have been widely studied in dry eye for over a decade $e^{20,21}$ and increasingly so with the advent of multiplex bead assays which allow many cytokines to be more quickly analyzed. ${ }^{5}$ Most of the research focuses on finding biomarkers for dry eye disease ${ }^{22}$ and for subtypes like aqueous deficient dry eye and autoimmune diseases like Sjögren's syndrome. Past studies suggest that IPL influence on cytokines may be responsible for some of its therapeutic efficacy. ${ }^{7}$

Transforming growth factor-beta (TGF- $\beta$ ), a growth cytokine with both anti-inflammatory and proinflammatory properties, ${ }^{23,24}$ was first associated with IPL when a 2013 dermatologic study of 20 patients revealed consistent upregulation of epidermal TGF- $\beta 1$ in skin biopsies 1 week after receiving IPL for inflammatory acne vulgaris $(p=0.007){ }^{25}$ TGF- $\beta 1$ has been found in the tear film and has been shown to be elevated in Sjögren's syndrome. ${ }^{26}$ One group found increased levels of TGF- $\beta 1$ after azithromycin in meibomian gland disease. ${ }^{27}$ However, no studies to date have examined association of TGF- $\beta$ and IPL in ocular rosacea and dry eye. Given the reported effect on 
skin in acne, TGF- $\beta$ was selected for the focus of this study. The amount of fluid needed for this assay is greater as compared to many other cytokine panels. Therefore, no other cytokines were included in this pilot study.

\section{Ocular Microbiome}

The ocular microbiome is a relatively small population, with roughly $1 / 100$ th the number of micro-organisms living on human skin. ${ }^{28}$ Culture-based microbiome analysis has found a dominance of gram-positive bacteria in the eye, such as Staphylococcus, Streptococcus, Corynebacterium, and Propionibacterium. ${ }^{29,30}$ The recent introduction of $16 \mathrm{~S}$ ribosomal ribonucleic acid (rRNA) gene metagenomics provides potential for a much more detailed and less biased understanding of the ocular microbiome compared to traditional culture methods. A study at the University of Washington analyzed ocular, buccal, and facial cutaneous swabs from 107 healthy volunteers using $16 \mathrm{~S}$ rRNA metagenomics, and found a predominance of Corynebacteria, Propionibacteria, and coagulase-negative Staphylococci in conjunctival swabs (consistent with prior culture-based findings). Additionally, they found that the ocular surface microbial community was found to be distinct from the skin and buccal microbiomes, with much greater diversity but lower total microbial quantity. ${ }^{31}$ Similar to the understanding that an unbalanced gut microbiome can inflict gastrointestinal damage, it is thought that an unbalanced ocular microbiome may contribute to pathologies such as endophthalmitis, blepharitis, or dry eye disease. ${ }^{28}$ A 2007 study performed 16S rRNA gene analysis on conjunctival swabs from patients with dry eye compared to healthy controls ${ }^{32}$ and found the microbiota of dry eye patients to have elevated bacterial counts as well as the presence of virulent bacteria that were not observed in healthy controls (namely Bacillus and Klebsiella). It is speculated that IPL may have direct antimicrobial effects in addition to anti-inflammatory effects, but there are no published studies to date that have further investigated this hypothesis.

The current study was designed to investigate the efficacy and mechanism of IPL in ocular rosacea with dry eye symptoms by analyzing TGF- $\beta$ and ocular microbiome in patients receiving IPL with MGX compared to MGX alone.

\section{Materials and Methods}

Twenty patients with a greater than 1 year history of ocular rosacea with MGD and DED presenting to the study clinician at Mayo Clinic Department of Ophthalmology in Scottsdale, Arizona, were recruited from November 2017 through September 2018. All patients meeting study criteria were consented and enrolled in a consecutive order. Participants were excluded based on the following factors: dry eye symptoms not alleviated with topical anesthetic (indicating possible neuropathic etiology), presence of systemic conditions including Sjögren's syndrome, graft versus host disease, StevensJohnson syndrome, presence or history of alkali burns, contact lens use, $>50 \%$ meibomian gland atrophy, Fitzpatrick $^{33}$ skin type $>$ IV, active periocular skin lesions or skin cancer, LASIK or other eye surgery within the past 12 months, and/or initiation of new DED treatment within the past 6 months. Those receiving treatment that started at least 6 months prior to enrollment, including systemic and/ or topical treatments for dry eye, were required to continue on the same dose without adding any new therapies until the end of the study. Research was approved by the Mayo Clinic Institutional Review Board (ID 16-008492) and followed the Declaration of Helsinki. The study was registered as a clinical trial on clinicaltrials.gov on Aug 17, 2017 (NCT03194698). Informed, written consent was obtained from all participants after a discussion of the risks, benefits, and patients' rights with the study clinician.

Ten patients were randomized to the control group (MGX), in which they received MGX every 4-6 weeks for four total treatments. Ten patients were randomized to the treatment group (IPL-MGX), in which they received IPL followed by MGX every 4-6 weeks for four total treatments. MGX and IPL were performed in accordance with the protocol previously published in detail ${ }^{11}$ which was originally developed by Rolando Toyos. 9,11 All subjects were instructed to use tobramycin/dexamethasone eye drops twice daily for 2 days following each appointment.

Laboratory staff and statisticians were masked to treatment groups. Data collected at the first visit prior to initiating treatment were used as a baseline for each participant. Visits 1 (baseline), 2, 3, and 4 (final) each included Ocular Surface Disease Index (OSDI) patient questionnaires $^{34}$ for subjective evaluation of patientreported symptoms, conjunctival injection score, lissamine green staining of interpalpebral conjunctiva, fluorescein corneal staining, Schirmer's 5-minute test with anesthesia, tear osmolarity, tear breakup time (TBUT), Snellen visual acuity, and tonometry (to monitor for adverse events such as cataract or glaucoma). Appointments 1 and 4 included additional evaluation; meibography was used to assess 
meibomian gland atrophy (OCULUS Keratograph 5M, Menlo Park, CA), Korb meibomian gland evaluator (Johnson \& Johnson, New Brunswick, NJ) was used to determine the number of meibomian glands open and the quality of meibum, and Lipiview tear film interferometry was used to determine lipid tear film thickness (Johnson \& Johnson, New Brunswick, NJ).

Additionally, at the beginning of visits 1 and 4 , $40 \mu \mathrm{L}$ of tears from each eye were collected from the surface of the palpebral conjunctiva near the caruncle using a capillary tube. Upper and lower eyelid swabs were collected from the eyelid margin orifices of meibomian glands after MGX treatment. Sterilized nitrocellulose collection paper was then placed on the anesthetized temporal conjunctiva and peeled off, collecting a superficial layer of conjunctival epithelium for impression cytology sampling. No saline, embedding medium, or preservative were added to any of the specimens. Sterile $1.5 \mathrm{~mL}$ safelock tubes (Eppendorf, Hamburg, Germany) were used. Biospecimens were stored at $-80^{\circ} \mathrm{C}$.

Tear samples were shipped to Eve Technologies Corporation (Calgary, Alberta), where Luminex ${ }^{\mathrm{TM}} 100$ xMAP technology (Luminex, Austin, TX, USA) was used for multiplexed quantification of TGF- $\beta$ in human tears following the MILLIPLEX TGF- $\beta$ 1,2,3 kit protocol (Millipore, St. Charles, MO, USA). Eyelid swab and impression cytology samples were shipped to Arizona State University for microbiome analysis using DNeasy PowerSoil Kit (QIAGEN) and next-generation sequencing in a MiSeq Illumina platform following the protocol by the Earth Microbiome Project. ${ }^{35}$ Mayo Clinic Rochester Bioinformatics then assigned taxonomy using DAD2 with SortMeRNA and the Silva v132 database. Microbiome data were reported as proportion of the total microbial population in order to offer a more understandable unit of measurement than quantifying actual microbial loads.

Wilcoxon Rank Sum and Sign-Rank tests were used to examine changes from baseline between treatment groups. Analyses were performed in SAS version 9.4 (SAS Institute Inc; Cary, North Carolina). Analysis followed an intention-to-treat approach.

\section{Results}

Twenty patients (40 eyes) were enrolled, and all patients completed the study. Slit-lamp examination and tonometry were performed at each visit to monitor for complications, and no individuals in either the MGX or IPL-MGX groups experienced any changes in intraocular pressure, visual acuity, lens findings, or other adverse events. The mean patient age was 59.9 years (range, 38-83), 85\% were female, and $90 \%$ were white. The mean age of the IPL/ MGX cohort was 59.3 years (range 39.0 - 83.0) and MGX cohort was was 60.5 years (range 38.0 - 83.0) $(\mathrm{p}=0.518)$. No patients were excluded due to Fitzpatrick skin type.

Mean baseline OSDI scoring was equivalent between IPL-MGX and MGX treatment groups (55.7 vs 43.5, $\mathrm{p}=0.212$ ). By the fourth visit, OSDI scores were significantly improved from baseline in the IPL-MGX treatment group compared to MGX alone ( $p=0.030)$. The number of meibomian glands yielding liquid secretions increased from baseline to visit 4 in both groups. The increase was greater in the IPL-MGX group, though not different enough to achieve statistical significance in this small cohort. No significant difference was detected between the two groups in fluorescein staining, lissamine green staining, bulbar redness score, Schirmer's testing, or percentage of meibomian gland atrophy measured by infrared meibography.

There was no statistically significant change in TGF$\beta 1,2$, or 3 concentration between groups $(p=0.385,0.709$, 0.948 , respectively) (Table 1$)$. Microbiome analysis at the genus level revealed a statistically significant reduction from baseline in Clostridium $(\mathrm{p}=0.001)$, Klebsiella $(\mathrm{p}=0.038), \quad$ Brevibacterium $\quad(\mathrm{p}=0.017), \quad$ Lactobacillus $(\mathrm{p}=0.010)$, Neisseria $(\mathrm{p}=0.027)$, Streptococcus $(\mathrm{p}=0.016)$, Corynebacterium $(\mathrm{p}=0.040)$, Butyricicoccus $(\mathrm{p}=0.011)$, and Actinomyces $(\mathrm{p}=0.001)$ in both MGX and IPL-MGX groups. However, there was no significant difference in these observed microbial changes between the two treatment groups.

\section{Discussion}

In this pilot study of IPL-MGX compared to MGX alone, patients who received IPL-MGX had significantly greater improvement in symptoms as measured by OSDI scores, which is in line with other literature on IPL for dry eye in ocular rosacea. This study revealed no significant change in tear TGF- $\beta$ concentration in either group. All patients experienced significant reduction in the quantity of several virulent bacteria, but with no statistically significant difference between treatment groups.

Tear cytokine analysis is a relatively novel field of dry eye research. Previous tear cytokine studies have observed elevated TGF- $\beta 1$ activity in dry eye, with the highest bioactivity in those with Sjögren syndrome. ${ }^{36}$ Another 
Table I Transforming Growth Factor- $\beta$ Change from Baseline

\begin{tabular}{|c|c|c|c|c|}
\hline & IPL-MGX $(\mathrm{N}=20)$ & $\operatorname{MGX}(\mathrm{N}=20)$ & Total $(\mathrm{N}=40)$ & p-value \\
\hline $\begin{array}{l}\text { TGF- } \boldsymbol{\beta} \text { I Change from Baseline } \\
\mathrm{N} \\
\text { Mean (SD) } \\
\text { Median } \\
\text { Range }\end{array}$ & $\begin{array}{l}10 \\
7.4(29.3) \\
4.8 \\
(-30.4-56.1)\end{array}$ & $\begin{array}{l}10 \\
-13.3(35.3) \\
-2.2 \\
(-100.1-21.0)\end{array}$ & $\begin{array}{l}20 \\
-3.0(33.3) \\
-0.2 \\
(-100.1-56.1)\end{array}$ & 0.385 \\
\hline $\begin{array}{l}\text { TGF- } \beta 2 \text { Change from Baseline } \\
\mathrm{N} \\
\text { Mean (SD) } \\
\text { Median } \\
\text { Range }\end{array}$ & $\begin{array}{l}16 \\
-466.3(917.9) \\
-469.8 \\
(-1832.8-962.4)\end{array}$ & $\begin{array}{l}13 \\
-410.8(1277.7) \\
-307 \\
(-3620.6-1472.6)\end{array}$ & $\begin{array}{l}29 \\
-44 I .4(1073.2) \\
-423.4 \\
(-3620.6-1472.6)\end{array}$ & 0.709 \\
\hline $\begin{array}{l}\text { TGF- } \beta 3 \text { Change from Baseline } \\
\mathrm{N} \\
\text { Mean (SD) } \\
\text { Median } \\
\text { Range }\end{array}$ & $\begin{array}{l}16 \\
-2.9(9.5) \\
0.1 \\
(-20.0-9.5)\end{array}$ & $\begin{array}{l}13 \\
-1.7(12.2) \\
-4.5 \\
(-17.4-21.1)\end{array}$ & $\begin{array}{l}29 \\
-2.4(10.6) \\
-3.2 \\
(-20.0-21.1)\end{array}$ & 0.948 \\
\hline
\end{tabular}

Notes: Tear concentration of transforming growth factor - beta (TGF- $\beta$ ) at baseline, and change in concentration from baseline to visit 4 . There was a mean decrease in concentration of all subtypes of TGF- $\beta$ in both treatment groups, except for TGF- $\beta$ I upregulation in the IPL-MGX group. Red = negative change, Black $=$ positive change, Units $=p g / m L$.

recent study observed that disrupted TGF- $\beta$ signaling in CD4+T cells was associated with improvement of DED in mouse models. ${ }^{37}$ These two studies suggest a correlation between elevated TGF- $\beta 1$ activity and worsening dry eye symptoms. In contrast, dermatology studies of acne rosacea demonstrate upregulation of TGF- $\beta 1$ in skin biopsies after IPL treatment, ${ }^{18}$ with improvement in disease following treatment. The discrepancies in data on TGF- $\beta$ highlight the difficult nature of extracting information about cytokines in human tears and the need for additional research. Our pilot study did not find a significant relationship between TGF- $\beta 1,2$, or 3 and dry eye symptoms or treatment with IPL.

One hypothesis of the efficacy of IPL for dry eye symptoms in ocular rosacea is the possible antimicrobial impact of the pulsed light. Compositional changes in the ocular microbiome can significantly impact ocular surface disorders. ${ }^{38}$ To our knowledge, this is the first study investigating changes in the ocular microbiome after treatment with IPL. This pilot study does not support the hypothesis that improvements in dry eye symptoms after IPL-MGX are due to alterations in the ocular microbiome. However, the entire patient cohort did demonstrate significant reduction in virulent bacteria after treatment with IPL-MGX or MGX alone. Potential mechanisms for this observation include improved secretion of antimicrobial meibum following treatment with MGX, and effects of antibiotic and steroid eyedrops that were used in the office after MGX treatment (Tobramycin $0.3 \%$ with Dexamethasone $0.1 \%$ ). Ultimately, IPL treatment was not shown to have additional benefit over MGX alone in decreasing virulent bacteria in this small set of patients.

There are several limitations to the findings of this study. The study is weakened by its small sample size and has limited generalizability with a cohort of $85 \%$ female and $90 \%$ white non-Hispanic/Latino. The small sample size prevented stratification by factors such as age, previous dry eye treatments, and timing of ocular rosacea diagnosis. In addition, patients were not blinded to their assigned treatment groups nor was there a placebo IPL treatment. There are many confounding variables in patients with dry eye disease. This study sought not to eliminate these variables but instead to control for variation during the study period. The only variable that differed for each patient from beginning to end of the study was the application of IPL-MGX or MGX alone. All patients received post-treatment anti-inflammatory regimen of 2 days of tobramycin and dexamethasone at each of the 4 treatments. Although this intervention may very well have influenced inflammation and microbiome across the cohort, it does not impede our ability to compare findings between treatment groups as it was consistent amongst all patients. It could however be a possible explanation for why there was a decrease in virulent bacteria seen across the cohort. 
Regarding tear cytokine analysis, extracting a significant volume of tear fluid from patients with dry eye was not always possible, and the laboratories struggled to extract data from these small samples necessitating the exclusion of several samples from analysis and further lowering the study's power. While other tear cytokine studies have diluted tear samples, tears in this study were not diluted in order to maintain the integrity of the samples. Future investigations could consider utilizing different techniques such as Schirmer's test for tear extraction that may demonstrate superior yield.

Considering ocular microbiota, the initial study design involved analyzing both the eyelid margin swabs and impression cytology samples. Eyelid margin swabs yielded insufficient sample volume for microbiome analysis. Therefore, microbiome genomics of conjunctival epithelium samples was performed alone. By analyzing only conjunctival epithelium, a large portion of the ocular area was missed. Prior studies have suggested that superficial, light pressure ocular swabs yield residential and transient species of the surface, while deep swabs with greater pressure yield different species that are localized to the mucosal layers. ${ }^{19}$ Ocular organisms live on not only the conjunctiva but also the cornea, eyelids, and eyelashes, and future studies of ocular microbiome should prioritize obtaining a more thorough and representative cell collection, perhaps comparing eyelid margin swabs to impression cytology samples.

Refractory dry eye symptoms can be extremely debilitating, impacting quality of life. There is an array of treatments offered to dry eye patients, many of which are not covered by insurance and lack any guarantee of improvement. Understanding the mechanism of IPL on dry eye from ocular rosacea would both enable providers to identify patients as good candidates based on their individual traits and expand current knowledge of dry eye thus opening the door to investigating additional therapeutic interventions.

\section{Conclusions}

This pilot study confirms that IPL-MGX for ocular rosacea patients with dry eye symptoms confers superior symptom relief compared to MGX alone. Future studies should consider investigating if IPL-MGX cotreatment may have an additive or synergistic affect that is not observed with IPL alone. No past studies have analyzed alterations in ocular TGF- $\beta$ or microbiome after treatment with IPL. Our study does not support the hypothesis that TGF- $\beta$ and/or microbiome alteration is the etiology of IPL's efficacy in treating dry eye from ocular rosacea. Prospective studies on IPLMGX with larger sample sizes are needed to further investigate tear cytokines and ocular microbiome in patients suffering from ocular rosacea with dry eye symptoms.

\section{Abbreviations}

IPL, intense pulsed light; MGX, meibomian gland expression; IPL-MGX, intense pulsed light with meibomian gland expression; MGD, meibomian gland disease; DED, dry eye disease; TGF- $\beta$, transforming growth factor-beta; OSDI, ocular surface disease index.

\section{Data Sharing Statement}

We will share deidentified microbiome sequencing data on the NIH Sequence Read Archive (https://www.ncbi.nlm.nih.gov/ sra). Deidentified cytokine data is available upon request.

\section{Acknowledgments}

The abstract of this paper was presented at the Association for Research in Vision and Ophthalmology (ARVO) virtual conference under the name "TGF $\beta 1$ and microbiome analysis following Intense Pulsed Light with Meibomian Gland Expression for dry eye symptoms from meibomian gland disease and ocular rosacea", as a poster presentation with interim findings. The poster's abstract was published in the journal of ARVO 2020, Invest Ophthalmol Vis Sci. 2020;61(7):104. The authors would like to acknowledge:

-Stephen Johnson at Mayo Clinic Arizona Department of Bioinformatics;

-Mayo Clinic Arizona Pathology Resource Core;

-Arizona State University Genomics Core;

-Andrews Family Foundation; and

-Research to Prevent Blindness.

-William Bourne MD

\section{Funding}

This study was supported by Mayo Clinic Internal Funding and received research funding from Ocugen Pharmaceuticals for Joanne F Shen.

\section{Disclosure}

The authors report no conflicts of interest in this work.

\section{References}

1. Dana R, Bradley JL, Guerin A, et al. Estimated prevalence and incidence of dry eye disease based on coding analysis of a large, all-age United States health care system. Am J Ophthalmol. 2019;202:47-54. doi:10.1016/j.ajo.2019.01.026 
2. Chew CK, Jansweijer C, Tiffany JM, Dikstein S, Bron AJ. An instrument for quantifying meibomian lipid on the lid margin: the Meibometer. Curr Eye Res. 1993;12(3):247-254. doi:10.3109/ 02713689308999470

3. Arita R, Morishige N, Fujii T, et al. Tear interferometric patterns reflect clinical tear dynamics in dry eye patients. Invest Ophthalmol Vis Sci. 2016;57(8):3928-3934. doi:10.1167/iovs.16-19788

4. Nelson JD, Shimazaki J, Benitez-del-Castillo JM, et al. The international workshop on meibomian gland dysfunction: report of the definition and classification subcommittee. Invest Ophthalmol Vis Sci. 2011;52(4):1930-1937. doi:10.1167/iovs.10-6997b

5. Bron AJ, de Paiva CS, Chauhan SK, et al. TFOS DEWS II pathophysiology report. Ocul Surf. 2017;15(3):438-510. doi:10.1016/j. jtos.2017.05.011

6. Jones L, Downie LE, Korb D, et al. TFOS DEWS II management and therapy report. Ocul Surf. 2017;15(3):575-628. doi:10.1016/j. jtos.2017.05.006

7. Liu R, Rong B, Tu P, et al. Analysis of cytokine levels in tears and clinical correlations after intense pulsed light treating meibomian gland dysfunction. Am J Ophthalmol. 2017;183:81-90. doi:10.1016/ j.ajo.2017.08.021

8. Goldberg DJ. Current trends in intense pulsed light. J Clin Aesthet Dermatol. 2012;5(6):45-53

9. Toyos R, Mcgill W, Briscoe D. Intense pulsed light treatment for dry eye disease due to meibomian gland dysfunction; a 3-year retrospective study. Photomed Laser Surg. 2015;33(1):41-46. doi:10.1089/pho.2014.3819

10. Gupta PK, Vora GK, Matossian C, Kim M, Stinnett S. Outcomes of intense pulsed light therapy for treatment of evaporative dry eye disease. Can J Ophthalmol. 2016;51(4):249-253. doi:10.1016/j. jcjo.2016.01.005

11. Vegunta S, Patel D, Shen JF. Combination therapy of intense pulsed light therapy and meibomian gland expression (IPL/MGX) can improve dry eye symptoms and meibomian gland function in patients with refractory dry eye: a retrospective analysis. Cornea. 2016;35 (3):318-322. doi:10.1097/ICO.0000000000000735

12. Vora GK, Gupta PK. Intense pulsed light therapy for the treatment of evaporative dry eye disease. Curr Opin Ophthalmol. 2015;26 (4):314-318. doi:10.1097/ICU.0000000000000166

13. Arita R, Mizoguchi T, Fukuoka S, Morishige N. Multicenter study of intense pulsed light therapy for patients with refractory meibomian gland dysfunction. Cornea. 2018;37(12):1566-1571. doi:10.1097/ ICO.0000000000001687

14. Dell SJ. Intense pulsed light for evaporative dry eye disease. Clin Ophthalmol (Auckland, NZ). 2017;11:1167-1173. doi:10.2147/ OPTH.S139894

15. Dell SJ, Gaster RN, Barbarino SC, Cunningham DN. Prospective evaluation of intense pulsed light and meibomian gland expression efficacy on relieving signs and symptoms of dry eye disease due to meibomian gland dysfunction. Clin Ophthalmol (Auckland, NZ). 2017;11:817-827. doi:10.2147/OPTH.S130706

16. Craig JP, Chen YH, Turnbull PRK. Prospective trial of intense pulsed light for the treatment of meibomian gland dysfunction. Invest Ophthalmol Vis Sci. 2015;56(3):1965-1970. doi:10.1167/iovs.14-15764

17. Guilloto CS, Garcia MJL, Colmenero RE. Effect of pulsed laser light in patients with dry eye syndrome. Arch Soc Esp Oftalmol. 2017;92 (11):509-515. doi:10.1016/j.oftal.2016.12.018

18. Jiang X, Lv H, Song H, et al. Evaluation of the safety and effectiveness of intense pulsed light in the treatment of meibomian gland dysfunction. J Ophthalmol. 2016;2016:1910694. doi:10.1155/2016/ 1910694

19. Milner MS, Beckman KA, Luchs JI, et al. Dysfunctional tear syndrome: dry eye disease and associated tear film disorders - new strategies for diagnosis and treatment. Curr Opin Ophthalmol. 2017;27(Suppl1):3-47. doi:10.1097/01.icu.0000512373.81749.b7
20. Lam H, Bleiden L, de Paiva CS, Farley W, Stern ME, Pflugfelder SC. Tear cytokine profiles in dysfunctional tear syndrome. Am $J$ Ophthalmol. 2009;147(2):198-205. e191. doi:10.1016/j. ajo.2008.08.032

21. Massingale ML, Li X, Vallabhajosyula M, Chen D, Wei Y, Asbell PA. Analysis of Inflammatory cytokines in the tears of dry eye patients. Cornea. 2009;28(9):1023-1027. doi:10.1097/ ICO.0b013e3181a16578

22. Jackson DC, Zeng W, Wong CY, et al. Tear interferon-gamma as a biomarker for evaporative dry eye disease. Invest Ophthalmol Vis Sci. 2016;57(11):4824-4830. doi:10.1167/iovs.16-19757

23. Massagué J. The transforming growth factor-beta family. Annu Rev Cell Biol. 1990;6:597-641. doi:10.1146/annurev.cb.06.110190.003121

24. Sporn MB, Roberts AB. Transforming growth factor- $\beta$ : multiple actions and potential clinical applications. JAMA. 1989;262 (7):938-941. doi:10.1001/jama.1989.03430070086036

25. Ali MM, Porter RM, Gonzalez ML. Intense pulsed light enhances transforming growth factor beta1/Smad3 signaling in acne-prone skin. J Cosmet Dermatol. 2013;12(3):195-203. doi:10.1111/jocd.12045

26. Pflugfelder SC, Jones D, Ji Z, Afonso A, Monroy D. Altered cytokine balance in the tear fluid and conjunctiva of patients with Sjögren's syndrome keratoconjunctivitis sicca. Curr Eye Res. 1999;19 (3):201-211. doi:10.1076/ceyr.19.3.201.5309

27. Zhang L, Su Z, Zhang Z, Lin J, Li D-Q, Pflugfelder SC. Effects of azithromycin on gene expression profiles of proinflammatory and anti-inflammatory mediators in the eyelid margin and conjunctiva of patients with meibomian gland disease. JAMA Ophthalmol. 2015;133(10):1117-1123. doi:10.1001/jamaophthalmol.2015.2326

28. Mukamal R. Microbiome of the eye. Am Acad Ophthalmol. 2019

29. Lu LJ, Liu J. Human microbiota and ophthalmic disease. Yale J Biol Med. 2016;89(3):325-330.

30. Miller D, Iovieno A. The role of microbial flora on the ocular surface. Curr Opin Allergy Clin Immunol. 2009;9(5):466-470. doi:10.1097/ ACI.0b013e3283303e1b

31. Doan T, Akileswaran L, Andersen D, et al. Paucibacterial microbiome and resident DNA virome of the healthy conjunctiva. Invest Ophthalmol Vis Sci. 2016;57(13):5116-5126. doi:10.1167/iovs.1619803

32. Graham JE, Moore JE, Jiru X, et al. Ocular pathogen or commensal: a PCR-based study of surface bacterial flora in normal and dry eyes. Invest Ophthalmol Vis Sci. 2007;48(12):5616-5623. doi:10.1167/ iovs.07-0588

33. Oakley A Fitzpatrick skin phototype DermNet NZ. 2012.

34. Real D, Hwang F, Bunya V. Dry eye syndrome questionnaires. Am Acad Ophthalmol. 2020.

35. EMP Ontology (EMPO). Earth microbiome project; 2020. Available from: https://earthmicrobiome.org/protocols-and-standards/empo/. Accessed January 05, 2021

36. Rong B, Tang Y, Liu R, et al. Long-term effects of intense pulsed light combined with meibomian gland expression in the treatment of meibomian gland dysfunction. Photomed Laser Surg. 2018;36 (10):562-567. doi:10.1089/pho.2018.4499

37. Rong B, Tang Y, Tu P, et al. Intense pulsed light applied directly on eyelids combined with meibomian gland expression to treat meibomian gland dysfunction. Photomed Laser Surg. 2018;36(6):326-332. doi:10.1089/pho.2017.4402

38. Caporaso JG, Lauber CL, Walters WA, et al. Global patterns of $16 \mathrm{~S}$ rRNA diversity at a depth of millions of sequences per sample. Proc Natl Acad Sci. 2011;108(Supplement 1):4516-4522. doi:10.1073/ pnas. 1000080107 


\section{Publish your work in this journal}

Clinical Ophthalmology is an international, peer-reviewed journal covering all subspecialties within ophthalmology. Key topics include: Optometry; Visual science; Pharmacology and drug therapy in eye diseases; Basic Sciences; Primary and Secondary eye care; Patient Safety and Quality of Care Improvements. This journal is indexed on PubMed

Submit your manuscript here: https://www.dovepress.com/clinical-ophthalmology-journal
Central and CAS, and is the official journal of The Society of Clinical Ophthalmology (SCO). The manuscript management system is completely online and includes a very quick and fair peer-review system, which is all easy to use. Visit http://www.dovepress.com/ testimonials.php to read real quotes from published authors. 